\title{
Pathological findings in three cases of fungal endocarditis complicating open-heart surgery
}

\author{
E. M. MCCONNELL AND C. ROBERTS \\ From the Pathology Department, Sefton General Hospital, Liverpool
}

SYNOPSIS Three cases of fungal endocarditis are described, each following a homograft valve replacement for aortic stenosis. The causative organism was $C$. albicans. The characteristic findings included soft, easily detached vegetations consisting of fungal colonies, aneurysms, embolization of large vessels, and lack of response to current therapeutic measures. The source of the infection is obscure.

Fungal endocarditis was first adequately described by Joachim and Polayes (1940) in a drug addict with aortic stenosis, following self-administered intravenous morphine and heroin. In 1958 Merchant and his colleagues (Merchant, Louria, Geisler, Edgcomb, and Utz, 1958) collected 34 cases of mycotic endocarditis from the literature. Eleven cases were caused by the Candida species, and Blastomyces ( 4 cases), Cryptococcus (1 case), Histoplasma (11 cases), Coccidiodes ( 2 cases), Aspergillus (4 cases), and Mucor (1 case) accounted for the remainder. Most of the patients were severely debilitated; five were known morphine addicts, eight had received long-term antibiotics, and in nine the endocarditis was secondary to a generalized or pulmonary infection.

The first recorded case of Candida endocarditis following open-heart surgery was reported by Koelle and Pastor (1956). There are now 19 recorded cases of fungal endocarditis complicating open-heart surgery, 17 of which were reviewed by Climie and Rachmaninoff (1965), who added two further cases of their own. The infection was due to Candida albicans in 10 cases, other Candida species in seven, and Aspergillus and Paecilomyces in one case each.

The present paper describes the pathological features of three further cases of this rare form of endocarditis as the lesion may be easily overlooked. The detailed clinical features and investigations will be the subject of a separate paper by other authors.

\section{CASE REPORTS}

CASE 1 C.B., a man aged 53 years, became febrile shortly after aortic valve homograft replacement for

Received for publication 12 January 1967. aortic stenosis. A searching investigation of the fever, including repeated blood cultures, did not reveal its cause. Two months after the operation, a saddle embolus lodged near the bifurcation of the aorta into the common iliac arteries, producing early ischaemic changes in the lower limbs. Embolectomy was performed, and culture of the specimen, a soft, friable thrombus, $3 \mathrm{~cm}$. diameter, yielded a growth of $C$. albicans. This was initially thought to be a contaminant and histologically the specimen was at first considered to be a fibrinous clot. The use of additional staining methods (periodic-acid-Schiff, Gram, and methenamine silver), however, revealed a mass of fungal colonies (Fig. 1) and a diagnosis of fungal endo carditis was made. Treatment with amphoteracin B was initiated but the patient's condition continued to deteriorate and he died three months after open-heart surgery.

Necropsy findings Externally the operation scars were well healed. Gangrene was present in three toes.

Internally, there were dense adhesions between the posterior aspect of the sternum, the anterior wall of the ascending aorta, and the two pericardial layers with small areas of encysted gelatinous effusions.

The heart was considerably increased in size due to concentric left ventricle wall hypertrophy. The surgical incision in the anterior wall of the left ventricle and the upper suture of the aortic valve homograft were intact.

The aortic valve was completely obscured on its ventricular surface by bulky, soft, smooth, friable vegetations making it impossible to identify the lower aortic suture lines (Fig. 2). The vegetations had spread onto the aortic aspect of the non-coronary aortic cusp and filled the corresponding sinus.

The myocardium of the left ventricle contained an extensive area of recent infarction, in addition to a smaller fibrosed infarct.

The coronary arteries were healthy, and the infarction was attributed to a mycotic embolus.

The orifice of the superior mesenteric artery was obliterated by a piece of vegetation, similar to that on the aortic valve. At the aortic embolectomy site there was a 


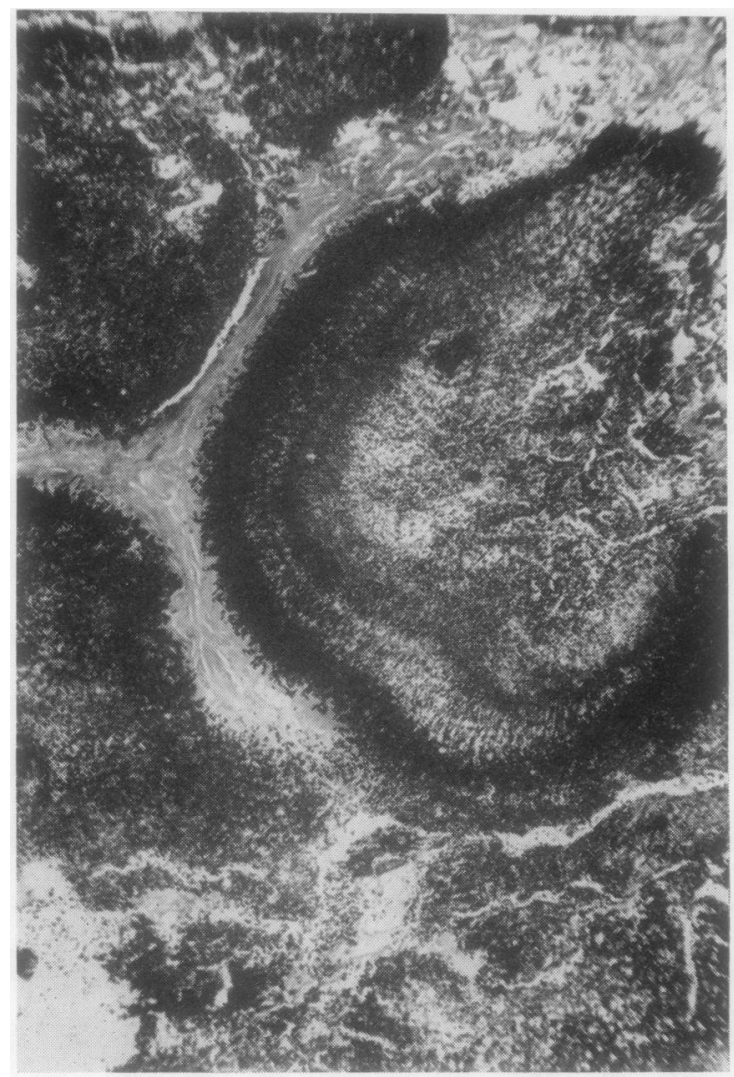

FIG. 1. Case 1. A section from the thrombus removed at embolectomy showing the characteristic fungal colonies with the darker staining advancing edge of budding cells. (Methenamine-silver stain $\times 30$.)

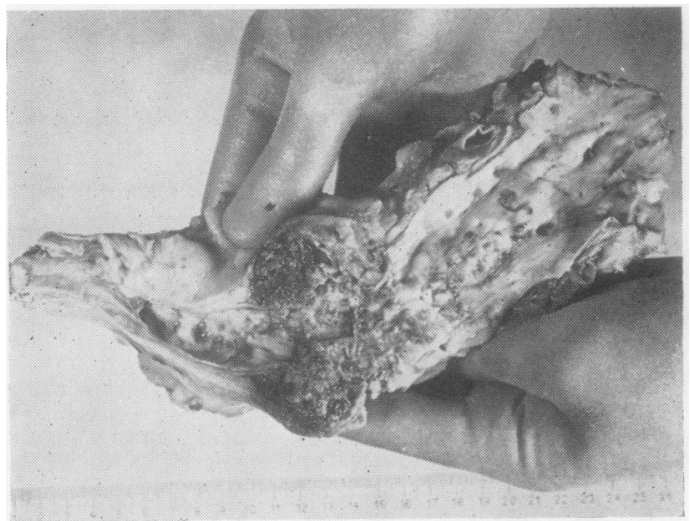

FIG. 3. Case 1. The abdominal aorta displaying the aneurysm containing blood clot and friable vegetations, which almost totally obliterated the lumen of the aorta.

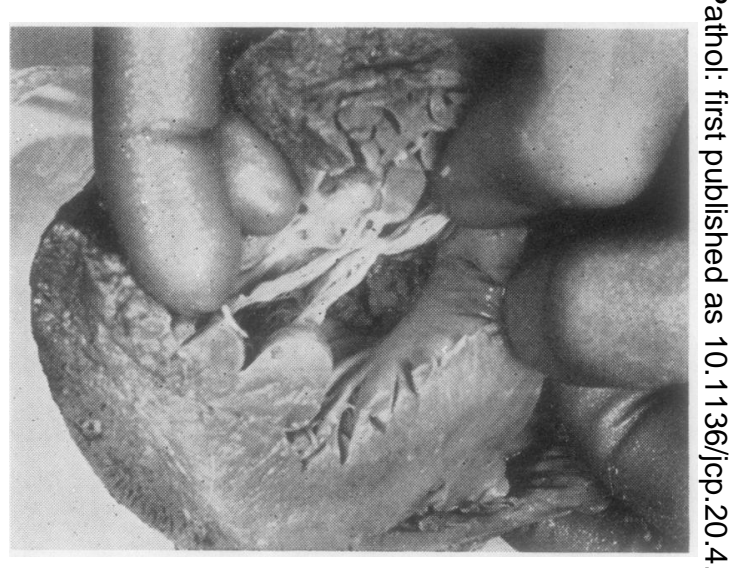

FIG. 2. Case 1. Inferior surface of the aortic valve. The chordae tendinae are displaced to one side showing the or bulky friable vegetations which completely obscured the 은 valve. The hypertrophy of the left ventricle and papillary $\rightarrow$ muscle is evident.

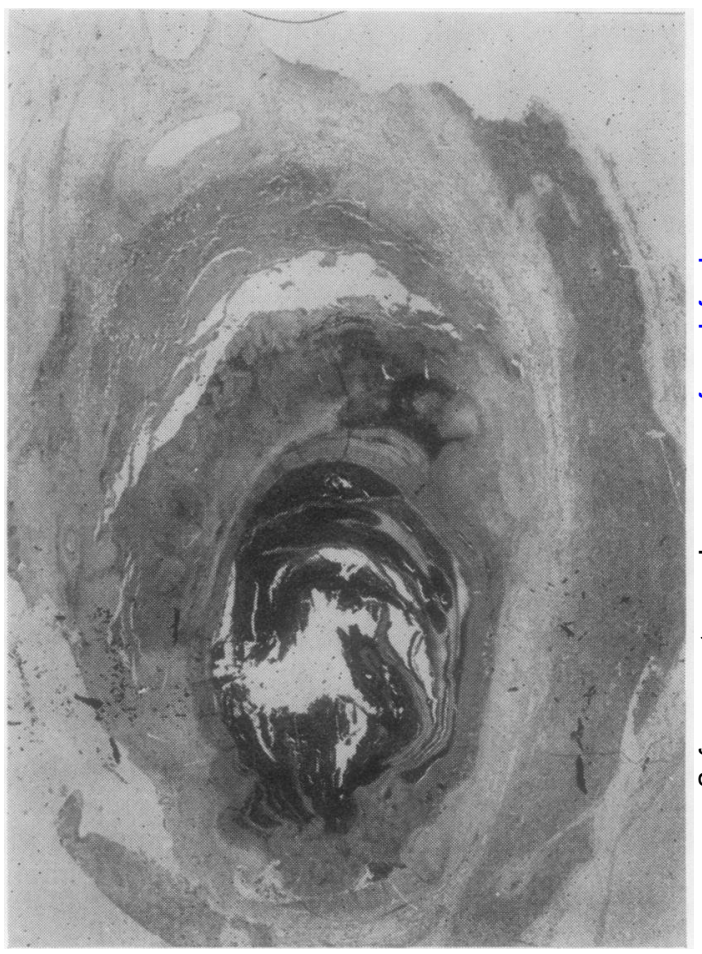

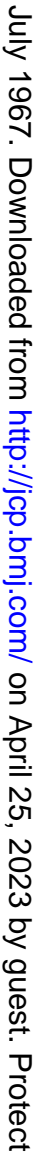

FIG. 4. Case 1. Cross section of the common iliac artery, $\vec{\otimes}$ into the lumen of which the vegetations and blood clot have $\sigma$ spread from the aortic aneurysm and have considerably reduced the lumen of the artery. 


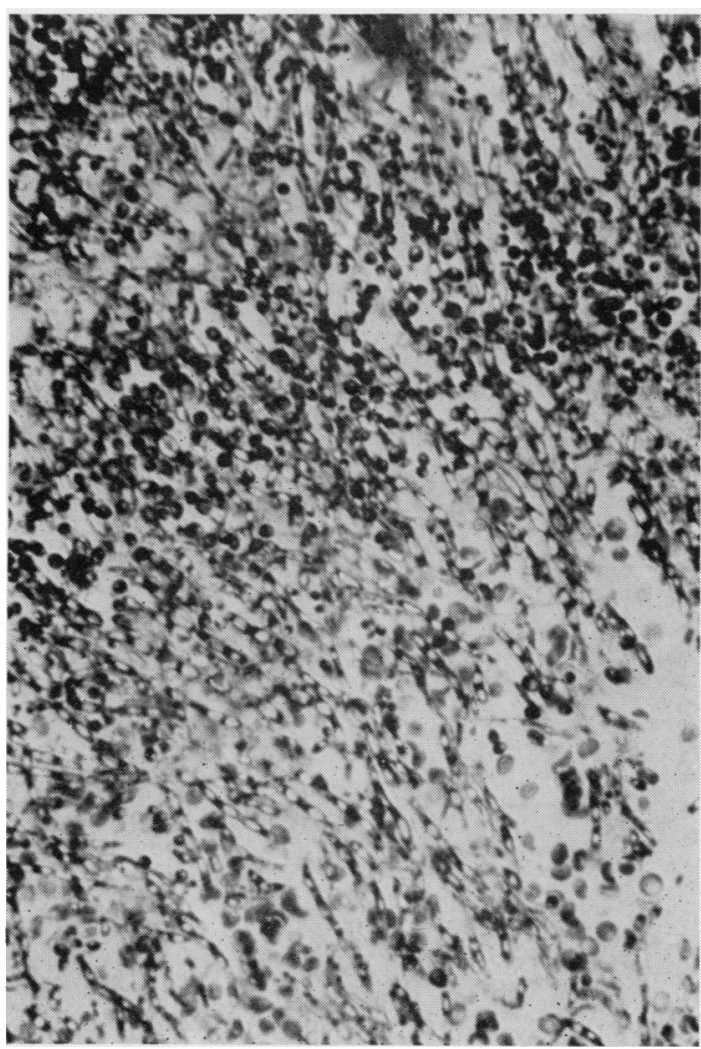

FIG. 5. Case 1. Section from the vegetations on the aortic valve showing hyphae with alternate light and dark bands and aggregations of fruiting bodies. (Methanamine-silver stain $\times$ 400.)

large leaking aneurysm, which extended along the proximal parts of the common iliac arteries (Figs. 3 and 4). Large, soft, friable vegetations and blood clot had almost completely obliterated the lumen of this part of the aorta and of the aneurysm and were protruding through the anterior aspect of the aneurysm.

The kidneys had a 'flea-bitten' appearance, due to numerous petechial haemorrhages in the cortex.

The spleen was enlarged $(\times 6)$ and contained areas of infarction.

The brain was not examined.

Culture of the aortic valve vegetation, the wall of the aneurysm and its contents on blood agar and Sabouraud's medium yielded a growth of $C$. albicans, identified by its morphological and cultural chracteristics.

Histologically the vegetations of the aortic valve and aneurysm were composed of fungal colonies (Fig. 5). There was an inflammatory response in the adjacent tissues with neutrophil infiltration, microabscess formation, and necrosis. Fungus was not demonstrated in the myocardial or splenic infarcts. The kidneys were too autolysed for valuable microscopic examination.
CASE 2 M.B., a man aged 37 years, became febrile three weeks after an aortic valve homograft had been performed, but extensive investigations, including repeated blood cultures, failed to reveal the cause of the pyrexia. He became apyrexial two weeks later, and was discharged home apparently well seven weeks after cardiac surgery.

At home he developed a thrombophlebitis in the calf, and was treated by his family doctor with a course of erythromycin. His condition failed to improve and two weeks after discharge, he was readmitted with the clinical features of bacterial endocarditis. A further 16 blood cultures were performed before penicillin and streptomycin therapy and one of these yielded $C$. albicans. Intravenous treatment with amphoteracin $B$ was given, but his condition deteriorated and he died 14 weeks after the aortic valve replacement.

Necropsy findings Permission for examination was limited to the heart. This was increased in size (weight $650 \mathrm{~g}$.), due mainly to left ventricle wall hypertrophy. The incision in the apex of the left ventricle and the upper suture line of the aortic valve homograft were intact.

The aortic valve was covered on its ventricular aspect by much soft, friable vegetation which extended onto the chordae tendinae and part of the papillary muscles. The lower aortic suture line was obscured by a $4 \mathrm{~cm}$. vegetation which continued over the valve.

The myocardium contained several areas of old fibrosis in the left ventricle wall.

The coronary arteries were normal.

Post-mortem culture on blood agar and Sabouraud's medium of the heart blood and aortic vegetation yielded a growth of $C$. albicans which was identified by its morphology and biochemical and cultural characteristics.

Histologically the aortic vegetations consisted of fungal colonies similar to those in case 1 , with a similar type of mycotic infiltration of the myocardium (Figs. 6 and 7). Again the surrounding tissues showed a neutrophil infiltration, microabscess formation, and necrosis.

CASE 3 J.C., a man aged 26 years, developed a persistent low-grade pyrexia after a valve homograft for aortic stenosis. This was initially considered to be due to the 'post-cardiotomy' syndrome, and he was treated with salicylates. Four weeks after the operation coagulasenegative staphylococci were grown from a blood culture and treatment was instituted with penicillin and later with erythromycin. His clinical condition, however, showed no improvement, and two weeks later $C$. albicans was cultured from a midstream specimen of urine. Further treatment with amphoteracin B reduced the pyrexia, but his general condition deteriorated and he died seven weeks after his operation.

Necropsy findings The degree of autolysis was greater than expected, due probably to a terminal $\mathrm{Cl}$. welchii infection. The sternum was adherent to the pericardium, and the pericardial cavity was completely obliterated by dense fibrous adhesions. Separation of the two pericardial layers revealed a false aneurysm $(4 \times 3 \mathrm{~cm}$.), anterior and to the left of the aorta.

The heart was considerably enlarged (weight $580 \mathrm{~g}$.) due to concentric left ventricle wall hypertrophy. The ventricular suture was intact. 


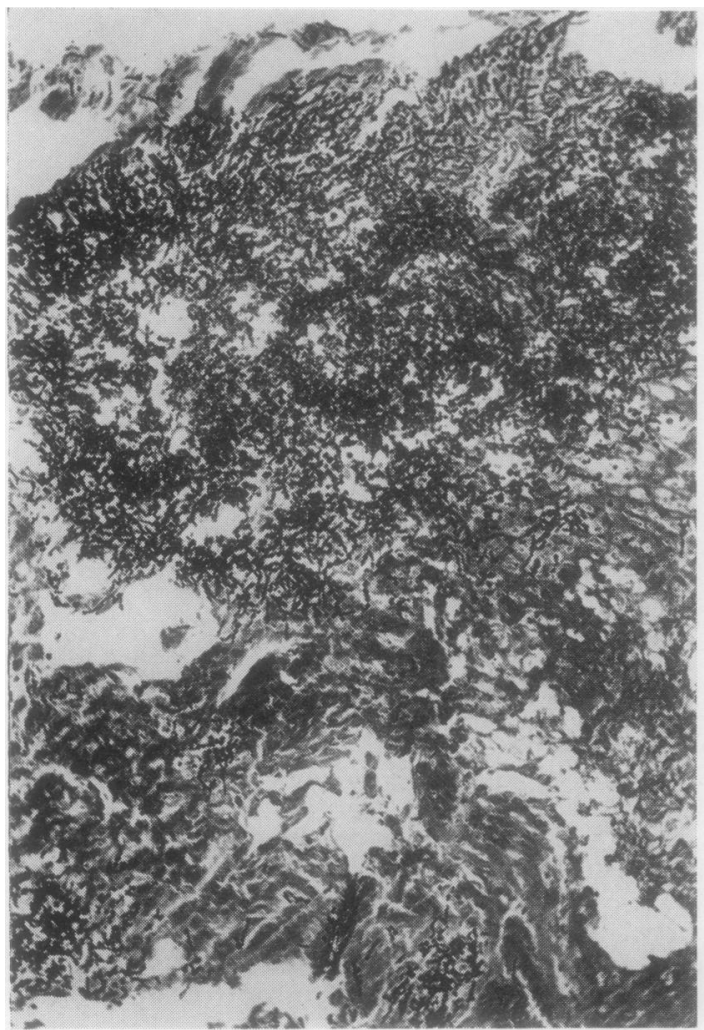

FIG. 6.

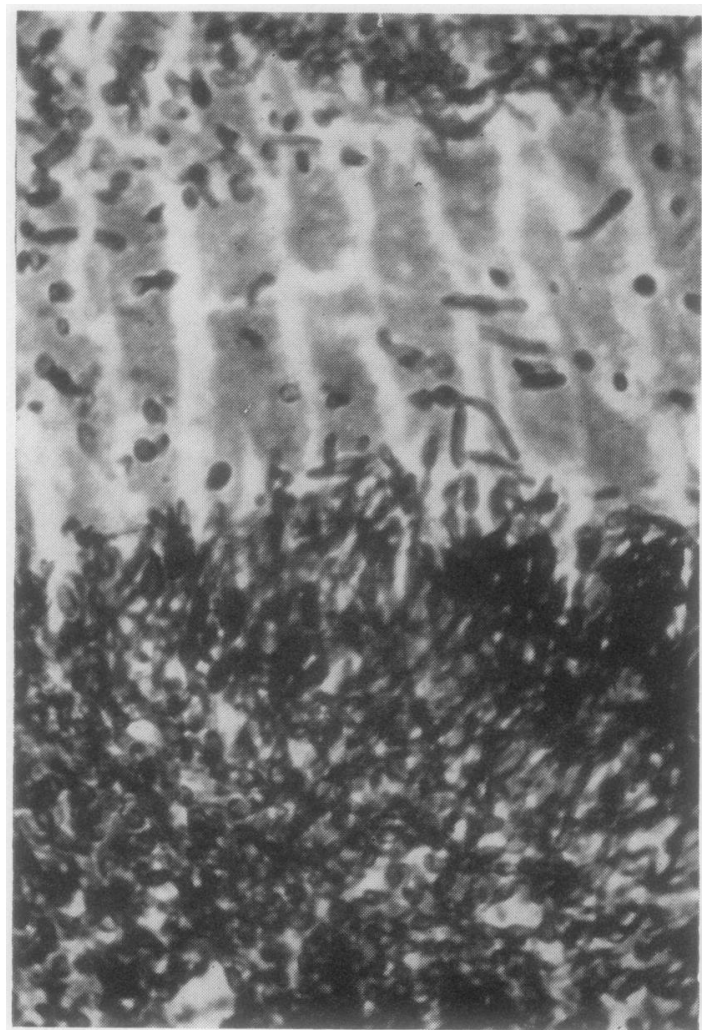

FIG. 7.

FIG. 6. Case 2. Infiltration of the myocardium of the left ventricle by Candida. (Methenamine silver stain $\times 90$.)

FIG. 7 Case 2. The edge of one of the colonies involving the myocardium showing the hyphae and small oval budding cells. (Methenamine silver $\times$ 400.)

The aortic valve was partly covered by friable vegetations, which replaced the left coronary cusp, filled the corresponding sinus, and continued into the false aneurysm. The latter was almost completely filled with blood clot and necrotic debris. The upper aortic suture line and the anterior and right coronary cusps and sinuses were normal.

The right coronary artery was normal. The orifice of the left coronary artery was not identified beneath the ulcerated necrotic tissue which filled the left coronary sinus, and its course through the false aneurysm was difficult to follow. The major branches of the left coronary artery were normal.

The spleen was enlarged $(\times 4)$ and contained several infarcts.

The kidneys contained several small infarcts.

The alimentary canal showed a severe enterocolitis.

The brain was normal except for one area of meningitis over the left temporal region.

C. albicans, which was cultured from the aortic vegetation, the aneurysm, the pericardium, the heart blood and the meninges, was identified by its morphology, biochemical and cultural characteristics, including the formation of chlamydospores on corn meal agar.
Histologically the aortic vegetation consisted of $\frac{\dot{0}}{7}$ fibrinoid material in which a few fungal colonies could be found. The aneurysm wall consisted of fibrinoid and $\delta$ necrotic material, in which fungi were scanty (Figs. 8 and 9). There was a neutrophil infiltration and fibrous prolifera- 0 ation in the adjacent friable tissues. Fungus was also identified on the pericardial aspect of the myocardium, but not in the renal or splenic infarcts. Amyloid material was present in the blood vessels and in the glomerular of and tubular basement membranes of the kidney as well as the blood vessels of the spleen. The connexion between this and the mycotic infection was obscure.

\section{DISCUSSION}

Post-operative bacterial endocarditis is well recog nized as an important complication of open heart surgery occurring in just under $10 \%$ of all cases (Denton, Pappas, Uricchis, Goldberg, and Likoff, 1957; Koiwai and Nahas, 1956). More recently fungal endocarditis, in particular that due to $C$. albicans, has also been recognized as a complication, 


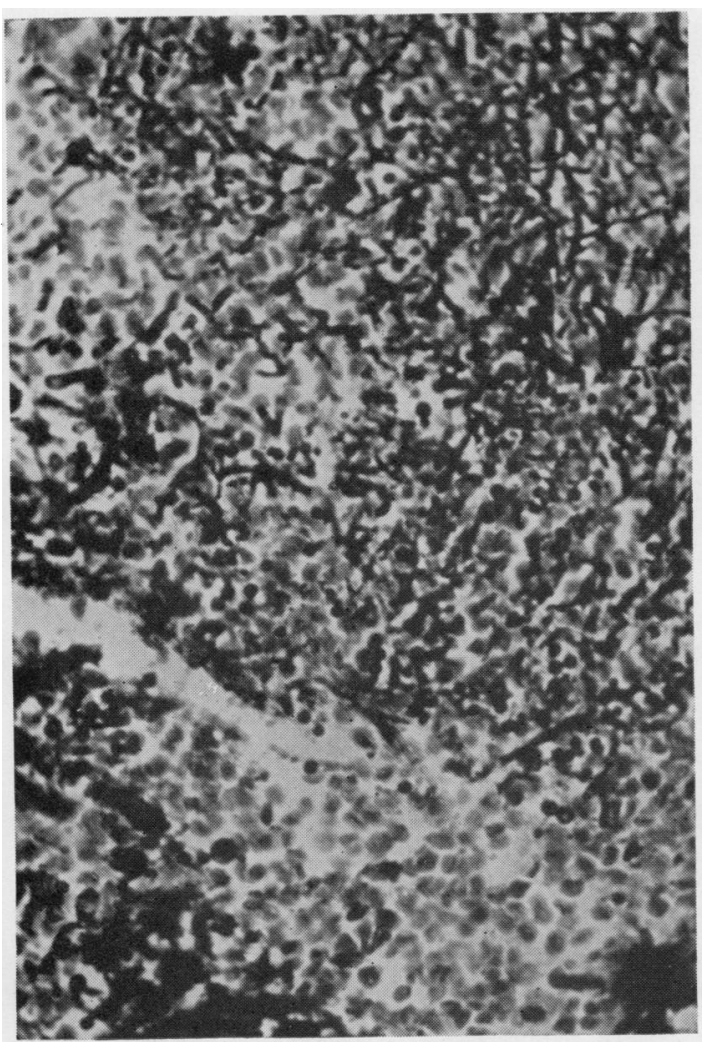

FIG. 8.

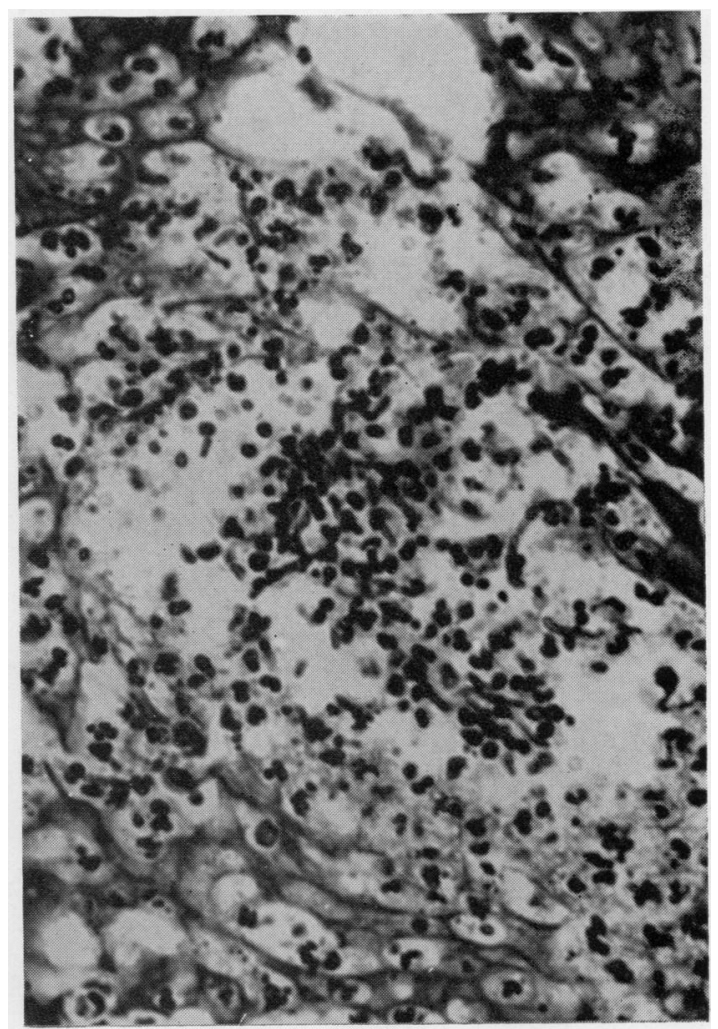

FIG. 9.

FIG. 8. Case 3. A section from the aortic aneurysm wall, showing the network of hyphae among the necrotic tissue. (Methenamine silver $\times$ 400.)

FIG. 9. Case 3. A small collection of heavily stained budding cells surrounded by a fibrous reaction from the base of the aorta. (Methenamine silver $\times 400$.)

and the present three cases bring the total number recorded to 22 .

The cases reported here confirm the clinical and pathological features described in previous cases. All three were adult males, continuing the male sex bias already noted (15 males, four females), with ages ranging from 26 to 53 years (previous average age 34 years).

The open-heart surgical treatment of aortic valve disease was followed, after an interval varying between a few days and one month, by a pyrexia, which was attributable to fungal endocarditis. The previous recorded cases had followed surgical treatment of aortic valve disease (nine cases), mitral valve disease (nine cases) and one case of ventricular septal defect. In these cases the interval between operation and the initial symptoms varied between 12 days and six months.

The clinical features were similar to those of bacterial endocarditis and the diagnosis of fungal endocarditis was made during life in case 1 by the recognition of the fungal nature of the thrombus removed at embolectomy, in case 2 by the identification of $C$. albicans from a blood culture, and in case 3 by the culture of the fungus from a midstream specimen of urine.

The embolization of a large vessel such as that which occurred in case 1 appears to be a distinguishing feature between bacterial and fungal endocarditis. This has been previously reported in 15 cases, in four of which the fungal nature of the thrombus was clearly recognized (Andriole, Kranetz, Roberts, and Utz, 1962; Jamshidi, Pope, and Friedman, 1963; Climie and Rachmaninoff, 1965).

Blood cultures yielded fungus in 14 of the 19 reported cases, but in many the reports were not available during life. In each of the present three cases $C$. albicans was cultured after death from the 
blood and aortic vegetation, and in addition in case 1 from the mycotic aneurysm and its contents and in case 3 from the myocardium and the meninges.

The source of the infection is a matter for conjecture. It is either introduced by some external agent, i.e., the replacement valves, the pump oxygenator, the infusion fluids, the intravenous catheters and needles, or it gains entrance to the bloodstream by virtue of its presence as a frequent member of the flora of the mouth and intestine. The present three cases occurred within a space of five months, suggesting some external factor rather than infection from the patients themselves.

Antibiotic therapy is considered by many to be a predisposing factor in the development of fungal endocarditis by destroying the intestinal bacterial flora, which would normally prevent fungal overgrowth. The mechanism of this and of the alteration of immunological defences to Candida infections by antibiotic therapy is the subject of an extensive review by Seelig (1966). The three cases under discussion received such therapy in keeping with current practice, which, in addition to routine operative prophylactic antibiotics, may include antibiotic cover for certain preoperative assessments, e.g., cardiac catheterization and dental treatment as in cases 1 and 3. Similar antibiotic therapy is also generally instituted in the treatment of subacute bacterial endocarditis.

The operative area in open-heart surgery seems to form a particularly suitable site for forming thrombus which becomes colonized by fungus, but cases of fungal endocarditis have also been reported following abdominal operations (Conn, Crean, McCabe, and MacLean, 1959; Louria and Dineen, 1960; Winner and Hurley, 1964) and peritoneal dialysis (Hurwich, 1966).

The growth of fungi from the blood, urine, and emboli in this type of case must always be regarded as significant and not rejected on the grounds of possible contamination of the specimen or culture medium, as we initially thought in case 1 .

The progression of the infection, in spite of treatment with the antifungal drugs at present $\stackrel{5}{\circ}$ available, is relentless, the average survival time from the date of heart surgery being under three months. Apparent cures of fungal endocarditis not associated with cardiac surgery have, however, been reported by several workers, including Kay, Bernstein, Feinstein, and Biddle (1961), who dealt with an affected cusp surgically, while the patients of Sanger, Taylor, Robicsek, Germuth, Senterfit, and McKinnon (1962) and Prinsloo and Pretorius (1966) responded to intravenous amphoteracin $\mathbf{B}$.

We wish to thank Dr. N. Coulshed and Dr. E. Epstein for permission to publish the clinical details, Mr. A. ir Scott for the histological preparation, and Mr. J. Brady Gr for the photographs.

\section{REFERENCES}

Andriole, V. T., Kravetz, H. M., Roberts, W. C., and Utz, J. P. (1962). Amer. J. Med., 32, 251.

Climie, A. R. W., and Rachmaninoff, N. (1965). J. thorac. cardiovasc. Surg., 50, 431.

Conn, N. K., Crean, G. P., MacCabe, A. F., and MacLean, N. (1959). Brit. med. J., 1, 944.

Denton, C., Pappas, E. G., Uricchio, J. F., Goldberg, H., and Likoff, W. (1957). Circulation, 15, 525.

Hurwich, B. J. (1966). Arch. intern. Med., 117, 405.

Jamshidi, A., Pope, R. H., and Friedman, N. H. (1963). Ibid., 112, 370.

Joachim, H., and Polayes, S. H. (1940). J. Amer. med. Ass., 115, 205.

Kay, J. H., Bernstein, S., Feinstein, D., and Biddle, M. (1961). New Engl. J. Med., 264, 907.

Koelle, W. A., and Pastor, B. H. (1956). Ibid., 255, 997.

Koiwai, E. K., and Nahas, H. C. (1956). Arch. Surg., 73, 272.

Louria, D. B., and Dineen, P. (1960). J. Amer. med. Ass., 174, 273.

Merchant, R. K., Louria, D. B., Geisler, P. H., Edgcomb, J. H., and Utz, J. P. (1958). Ann. intern. Med. 48, 242.

Persellin, R. H., Harding, O. M., and Lewis, F. J. (1961). Ibid., 54, 127.

Prinsloo, J. G., and Pretorius, P. J. (1966). Amer. J. Dis. Child., 111, 446.

Sanger, P. W., Taylor, F. H., Robicsek, F., Germuth, F., Senterfit, L. and McKinnon, G. (1962). J. Amer. med. Ass., 181, 88.

Seelig, M. S. (1966). Bact. Rev., 30, 442.

Winner, H. I., and Hurley, R. (1964). Candida albicans. Churchill, London. 